ISSN 0103-5150

Fisioter. Mov., Curitiba, v. 26, n. 3, p. 657-664, jul./set. 2013

Licenciado sob uma Licença Creative Commons

\title{
Efeitos da aplicação da corrente polarizada e da iontoforese na gordura localizada em mulheres $^{1}$
}

\author{
Effects of polarized current and iontophoresis \\ application in localized fat in women
}

\section{Ana Carolina Brandt de Macedo ${ }^{[a]}$, Francini Cunico ${ }^{[b]}$, Larissa Sassi ${ }^{[b]}$, Jocilene Albuquerque ${ }^{[b]}$, Fernanda Borges ${ }^{[b]}$}

[a] Fisioterapeuta, mestre em Engenharia Biomédica pela Universidade Tecnológica do Paraná (UTFPR), docente do curso de Fisioterapia da Universidade Tuiuti do Paraná (UTP), Curitiba, PR - Brasil, e-mail: acbrandt@bol.com.br

[b] Fisioterapeutas pela Universidade Tuiuti do Paraná (UTP), Curitiba, PR - Brasil, e-mails: francinicunico@gmail.com, larisassi@yahoo.com.br, jalbuqueque@gmail.com, borgesfer_@hotmail.com.br

\section{Resumo}

Introdução: As correntes polarizadas possuem efeito iônico e no polo negativo predomina o aumento do metabolismo local. Objetivo: Comparar os efeitos da aplicação das correntes polarizadas e da iontoforese na gordura localizada em flancos. Materiais e métodos: Foram selecionadas 24 voluntárias, com faixa etária 18-30 anos, sedentárias, sem antecedentes gestacionais e sem cirurgias em região abdominal. Foram divididas em quatro grupos: grupo $A(n=4)$, com aplicação de correntes polarizadas; grupo B $(n=6)$, iontoforese; grupo $C(n=6)$, cosmético diretamente sobre a pele; e grupo D $(n=5)$, controle. 0 protocolo das correntes consistiu em da aplicação das correntes galvânica, difásica, curto período e longo período, cada uma durante 5 minutos, sendo realizadas duas vezes por semana, totalizando dez sessões. Na iontoforese o produto utilizado foi a centelha asiática. A técnica de aplicação foi a transversal. Para avaliação e reavaliação,

1 Clinical Trial Registration Number ACTRN12610000255088. 
efetuou-se a adipometria e conferiram-se medida da circunferência, peso, altura e IMC. Resultados: Na comparação intergrupos, verificou-se que os grupos A e B não apresentaram diferenças significativas nas medidas de circunferência e em relação à adipometria somente o grupo B apresentou resultados significativos $(p<0,05)$. Na comparação intergrupos, o mesmo grupo obteve a maior significância. Conclusão: A associação de cosmético e corrente polarizada proporcionou a redução das medidas em flancos, o que ressalta os benefícios da iontoforese.

Palavras-chave: Estimulação elétrica transcutânea. Tecido adiposo. Iontoforese.

\section{Abstract}

Introduction: The polarized current has ionic effects and in the negative pole predominate the increase of local metabolism. Objective: To compare the effects of polarized current and iontophoresis in localized fat. Materials and methods: It was selected 24 volunteers, aged 18-30, sedentary, without any pregnancy and abdominal surgery. They were divided in four groups: group $1(n=4)$, application of current polarized; group 2 $(n=6)$, ionthophoresis, group $3(n=6)$, cosmetic application directly by skin; and group 4 , control $(n=5)$. The current protocol was the application of galvanic current, biphasic, short and long period, each for 5 minutes, and held two times per week, totaling ten sessions. In iontophoresis the product used as Centella asiatica, with negative polarity which was placed on the cathode. The application's technique was the transversal. To evaluate and review were conducted to adipometry, circumference, weight, height and BMI. Results: In comparison between $A$ and $B$ groups it was verified no statistic differences in measures of circumference; in adipometry, only group B showed significant results $(p<0.05)$. Conclusion: Association of cosmetic and polarized current provided the reduction of the measures in the flanks which highlights the benefits of iontophoresis.

Keywords: Transcutaneous electric nerve stimulation. Adipose tissue. Iontophoresis.

\section{Introdução}

A gordura localizada é uma hipertrofia das células adiposas uniloculares encontrada em uma região mais específica, principalmente na região dos flancos, determinando abaulamentos diversos em razão do volume existente. Essa hipertrofia é resistente a dietas, massagem e ginásticas $(1,2)$. Pesquisas revelam que o excesso de gordura localizada na região central do corpo, principalmente em abdômen, está associado a um número maior de problemas de saúde, como: hiperinsulinemia, hiperlipidemia, Diabetes mellitus tipo 2, hipertensão, gota e artrite (3).

A cirurgia plástica é normalmente o método mais procurado (2). Segundo estudo com 38 pacientes submetidos a cirurgia plástica, a região abdominal, culotes e flancos foram os mais apontados como problemáticos para a autoaceitação corporal feminina, motivando-as a se submeteram a lipoaspiração (4). Em um estudo retrospectivo, no qual foram investigados 38 procedimentos de cirurgia de lipoaspiração, concluiu-se que este é um método satisfatório e seguro para a remoção da gordura localizada (5).
Contudo, no mercado, existem outros tratamentos não invasivos, realizados por fisioterapeutas, como: drenagem linfática, termoterapia, crioterapia, massagem modeladora, eletroterapia (corrente russa, radiofrequência, corrente galvânica e diadinâmica) (6).

A aplicação das correntes polarizadas para tratamento de diversas patologias é utilizada desde a descoberta da eletricidade. Sabe-se que a polaridade fixa nas correntes pode trazer inúmeros benefícios como hidratação, aumento do aporte circulatório no polo negativo, bem como vasoconstrição e analgesia no polo positivo. Já a iontoforese aproveita as correntes polarizadas para a penetração de cosméticos que possuem substâncias polares $(6,7)$. As correntes galvânicas e diadinâmicas são utilizadas para realizar a iontoforese (8). Já existem vários estudos comprovando que a corrente polarizada pode auxiliar na penetração de produtos ionizados (9-11). Isso traz inúmeros benefícios ao paciente como aplicação localizada, diminuição da ação sistêmica do medicamento e diminuição de problemas gastrointestinais (12).

$\mathrm{Na}$ área da fisioterapia dermatofuncional, a iontoforese com cosméticos é usada como coadjuvante 
no tratamento da gordura localizada, lipodistrofia ginoide, cicatrizes hipertróficas e queloides (13). Em virtude da escassez de literatura sobre tratamentos para a gordura localizada, o objetivo do presente estudo foi comparar os efeitos da aplicação da corrente polarizada e da iontoforese no tratamento da gordura localizada em flancos em mulheres sedentárias.

\section{Materiais e métodos}

Quarenta voluntárias com idade entre 18 e 30 anos, sedentárias, apresentando depósito adiposo na região de flancos foram selecionadas, porém somente 21 concluíram o estudo. Os critérios de exclusão foram: praticar atividade física, ter filhos, ter se submetido a alguma cirurgia abdominal, e fazer algum tipo de dieta. Todas as voluntárias assinaram o Termo de Consentimento Livre e Esclarecido, previamente aprovado pelo Comitê de Ética e Pesquisa da Universidade Tuiuti do Paraná (UTP) sob o protocolo n. 042/2008.

Antes da aplicação do protocolo, as voluntárias foram submetidas a avaliação da composição corporal pelos seguintes protocolos: relação peso-altura (IMC) e medida das dobras cutâneas (adipometria) (6) do tríceps, suprailíaca e abdominal; também foram mensuradas as circunferências da região supraumbilical, infraumbilical e cintura. Para determinação da estatura, utilizou-se um estadiômetro com escala de medida de $0,1 \mathrm{~cm}$, enquanto para verificação do peso corporal foi empregada uma balança antropométrica com precisão de 100 $\mathrm{g}$, a partir do método apresentado por Gordon et al. (14). As circunferências foram realizadas mediante utilização de uma fita antropométrica de aço flexível com precisão de uma casa decimal, de acordo com as padronizações sugeridas por Callaway et al. (15).

A amostra foi dividida aleatoriamente em quatro grupos: A, B, C e D. 0 GA $(\mathrm{n}=4)$ foi submetido à aplicação das correntes polarizadas; o GB $(n=6)$ à iontoforese; o GC $(n=6)$ à aplicação direta sob a pele do cosmético; e o GD ( $n=5)$, controle. GA e GB foram submetidos a dez aplicações das correntes, duas vezes por semana. No GC, foram aplicados o cosmético duas vezes por semana durante cinco semanas e o GD, controle, não foi submetido a nenhum procedimento, sendo reavaliado após cinco semanas.

Para a aplicação das correntes, inicialmente efetuou-se aassepsia local com álcool 70\%. Dois eletrodos metálicos, com esponja umedecida com água destilada, foram posicionados de cada lado transversalmente em cada flanco, sendo os da região anterolateral do abdômen o polo negativo, e os outros, na região posterolateral do abdômen, o positivo. A técnica de eletrodos fixos foi determinada de acordo com o estudo de Ferreiraet al. (16), que observaram maior eficácia em relação aos móveis. Utilizou-se primeiramente a corrente difásica (DF) por 5 min., corrente que diminui a impedância dos tecidos e prepara-os para as outras correntes, seguida da corrente de curto período (CP) e longo período (LP), cada uma por $5 \mathrm{~min}$., para efeitos vasculares. Por último, aplicou-se a corrente galvânica (G), potencializando os efeitos iônicos das correntes polarizadas (17). A dosimetria aplicada foi de 0,15 $\mathrm{mA} / \mathrm{cm}^{2}$; como o eletrodo tinha uma área de $25 \mathrm{~cm}^{2}$, totalizou-se uma dose de 3,75 mA (8).

No GB, além da aplicação feita no GA, foi adicionado o produto composto de centelha asiática 5\% (13) com arnica (polaridade negativa), no eletrodo negativo (6). No outro eletrodo foi colocada água destilada. No GC, as voluntárias aplicaram o produto diretamente sobre a pele limpa, deixando-o penetrar totalmente. Após o término das dez sessões, as pacientes foram encaminhadas para uma reavaliação, para análise comparativa.

Para análise estatística dos resultados, foram utilizados os testes: teste $t$ de Student para analisar a diferença intragrupo referente às sessões iniciais e finais e ANOVA (Análise de variância) para realizar a comparação intergrupos, usando a diferença entre as sessões iniciais e finais.

\section{Resultados}

Os resultados são apresentados entre cada grupo. A Tabela 1 apresenta o peso das voluntárias a fim de se investigar a influência do mesmo na redução de medidas. Verifica-se que as voluntárias GA4, GA5 e GC6 apresentaram redução $\geq 1$ kg: e GA3, GC3, GC6, GD2 e GD6 tiveram aumento $\geq 1 \mathrm{~kg}$. Ressalta-se que todas foram orientadas a alimentar-se normalmente.

Os resultados apresentados na Tabela 2 , referentes ao GA, demonstram que a maioria das variáveis não obteve significância estatística. Apenas a variável circunferência infraumbilical apresentou significância $(\mathrm{p}=0,018)$.

Os resultados apresentados na Tabela 3, referentes ao GB, demonstram um efeito significativo na adipometria suprailíaca $(p=0,034)$ e na porcentagem de gordura ( $p=0,048)$, o qual foi reflexo do valor anterior. As demais variáveis não obtiveram significância estatística. 
Tabela 1 - Medidas do peso de todos os grupos

\begin{tabular}{lccccccccccc}
\hline & \multicolumn{3}{c}{ Grupo A } & \multicolumn{3}{c}{ Grupo B } & \multicolumn{3}{c}{ Grupo C } & \multicolumn{3}{c}{ Grupo D } \\
Voluntária & \multicolumn{2}{c}{ Peso } & \multicolumn{3}{c}{ Voluntária } & \multicolumn{2}{c}{ Peso } & Voluntária & Peso & Voluntária & Peso \\
& Antes & Depois & & Antes & Depois & & Antes & Depois & & Antes & Depois \\
\hline GA1 & 59,0 & 59,0 & GB1 & 50,0 & 50,0 & GC1 & 53,0 & 53,4 & GD1 & 53,0 & 54,0 \\
GA2 & 62,0 & 62,0 & GB2 & 54,0 & 53,7 & GC2 & 51,0 & 51,4 & GD2 & 56,0 & 56,0 \\
GA3 & 62,0 & 62,0 & GB3 & 57,0 & 57,0 & GC3 & 59,0 & 60,0 & GD3 & 53,0 & 53,0 \\
GA4 & 63,2 & 61,3 & GB4 & 58,0 & 58,0 & GC4 & 58,9 & 59,0 & GD4 & 57,0 & 57,0 \\
GA5 & & & GB5 & 59,7 & 59,0 & GC5 & 57,0 & 57,5 & GD5 & 52,0 & 53,0 \\
GA6 & & & GB6 & 56,2 & 55,5 & GC6 & 54,0 & 55,0 & GD6 & & \\
Média & 61,55 & 61,07 & Média & 55,81 & 55,53 & Média & 55,48 & 56,05 & Média & 54,2 & 54,6 \\
\hdashline d.p. & 1,79 & 1,42 & d.p. & 3,12 & 3,0 & d.p. & 3,02 & 3,05 & d.p. & 2,16 & 1,81 \\
\hline
\end{tabular}

Fonte: Dados da pesquisa.

Tabela 2 - Resultados da comparação antes e após aplicação das correntes polarizadas do GA ( $\mathrm{n}=6$ )

\begin{tabular}{lccc}
\hline Variáveis & $\begin{array}{c}\text { Antes } \\
\text { Média (d.p.) }\end{array}$ & $\begin{array}{c}\text { Depois } \\
\text { Média (d.p.) }\end{array}$ & p \\
\hline IMC & $23,05(0,9)$ & $22,72(0,49)$ & 0,303 \\
\hline Circunferência cintura & $78(5,35)$ & $76,75(4,5)$ & 0,278 \\
Circunferência supraumbilical & $79,75(5,05)$ & $78,37(4,19)$ & 0,391 \\
Circunferência infraumbilical & $85,5(3,69)$ & $83,25(3,77)$ & $0,018^{*}$ \\
\hline Adipometria abdomen & $41(6,63)$ & $40,75(6,5)$ & 0,900 \\
Adipometria suprailíaca & $31(6,37)$ & $29,25(5,67)$ & 0,188 \\
\hline \% gordura & $32,07(7,63)$ & $31,27(7,47)$ & 0,539 \\
\hline
\end{tabular}

Legenda: * $=$ significância estatística $(p<0,05)$.

Fonte: Dados da pesquisa.

Na Tabela 4 são descritos os resultados da comparação antes e após aplicação do cosmético, no GC. Observa-se que a variável que obteve significância estatística foi a circunferência infraumbilical $(p=0,005)$. Surpreendentemente, esse resultado ocorreu pelo aumento e não pela diminuição na medida dessa região. As demais variáveis não obtiveram significância estatística. 0 resultado também foi observado no grupo controle, ou GD.

Os resultados apresentados na Tabela 5 não apresentam significância em nenhuma das variáveis. Assim como explicado anteriormente para o GC, esses resultados demonstraram um aumento e não uma diminuição na medida dessas regiões. Como esperado, as demais variáveis não obtiveram significância estatística.

\section{Discussão}

Analisando os resultados do GA, foram observadas somente mudanças significativas nas medidas da circunferência infraumbilical. Segundo Milani et al. (13), os efeitos terapêuticos da corrente galvânica devem-se, em grande parte, aos efeitos polares da corrente contínua sobre as células do organismo. 
Tabela 3 - Resultados da comparação antes e após aplicação da iontoforese do GB ( $\mathrm{n}=6)$

\begin{tabular}{lccc}
\hline Variáveis & Antes & Depois & p \\
& Média (d.p.) & Média (d.p.) & 0,363 \\
\hline IMC & $21,78(2,03)$ & $21,68(1,99)$ & 0,167 \\
\hline Circunferência cintura & $70,42(3,75)$ & $69,42(3,95)$ & 0,129 \\
Circunferência Supra umbilical & $75,83(7,91)$ & $74,08(6,73)$ & 0,584 \\
Circunferência Infra umbilical & $81,58(6,58)$ & $81,17(6,37)$ & 0,081 \\
\hline Adipometria abdomen & $30,17(7,08)$ & $27,67(6,38)$ & $0,034^{*}$ \\
Adipometria supra-ilíaca & $24,17(5,85)$ & $17,83(4,02)$ & $0,048^{*}$ \\
\hline \% gordura & $28,97(4,44)$ & $26,27(3,58)$ & \\
\hline
\end{tabular}

Legenda: * $=$ significância estatística $(p<0,05)$.

Fonte: Dados da pesquisa.

Tabela 4 - Resultados da comparação antes e após aplicação do cosmético do GC ( $\mathrm{n}=6)$

\begin{tabular}{lccc}
\hline Variáveis & Antes & Depois & p \\
& Média (d.p.) & Média (d.p.) & 0,218 \\
\hline IMC & $21,88(2,62)$ & $22,12(2,87)$ & 0,611 \\
\hline Circunferência cintura & $70,67(6,53)$ & $70,83(6,65)$ & 0,135 \\
Circunferência supraumbilical & $74,83(6,97)$ & $76(6,96)$ & $0,005^{\star}$ \\
Circunferência infraumbilical & $82,08(7,88)$ & $83,67(8,07)$ & 0,889 \\
\hline Adipometria abdomen & $28,5(13,43)$ & $28,67(11,94)$ & 0,311 \\
Adipometria suprailíaca & $20,17(8,75)$ & $23,67(15,63)$ & 0,949 \\
\hline \% gordura & $27,33(6,84)$ & $27,4(6,60)$ & \\
\hline
\end{tabular}

Legenda: * $=$ significância estatística $(\mathrm{p}<0,05)$.

Fonte: Dados da pesquisa.

Segundo Gratieri et al. (6), esses efeitos poderiam promover um incremento na nutrição do tecido afetado consequente ao aumento da circulação local, que ocorre principalmente no nível do polo negativo, que é mais estimulante. Quanto às correntes diadinâmicas utilizadas, sabe-se que a DF é usada primeiramente para diminuir a impedância da pele. A CP e a LP possuem efeito vascular. Apesar dos efeitos iônicos causados pela aplicação dessas correntes, não houve influência na redução das medidas abdominais; esse resultado pode estar relacionado com a profundidade de aplicação, o qual não consegue atingir o tecido adiposo por se tratar de correntes de baixa frequência (18).
O GB apresentou alguns resultados significativos como na adipometria suprailíaca e na composição corporal. Segundo Guaratini et al. (8), a iontoforese é um procedimento de efeitos locais, mesmo que superficiais, como a ação localizada de medicamento, podendo estar em maior concentração na área a ser tratada. De fato, os resultados deste estudo confirmam isso, uma vez que os efeitos de redução de medida foram apenas locais, não havendo diminuição do IMC dos indivíduos. Os resultados apresentados também sugerem que o cosmético utilizado na iontoforese obteve um efeito importante na redução da gordura localizada na região 
Tabela 5 - Resultados do grupo $4(n=6)$

\begin{tabular}{lccc}
\hline Variáveis & $\begin{array}{c}\text { Antes } \\
\text { Média (d.p.) }\end{array}$ & $\begin{array}{c}\text { Depois } \\
\text { Média (d.p.) }\end{array}$ & p \\
\hline IMC & $24,46(5,19)$ & $24,94(5,93)$ & 0,283 \\
\hline Circunferência cintura & $73(5,52)$ & $73,8(6,14)$ & 0,090 \\
Circunferência supra-umbilical & $76,20(4,60)$ & $77(4,63)$ & 0,241 \\
Circunferência infra-umbilical & $81,20(2,04)$ & $82,4(2,96)$ & 0,070 \\
\hline Adipometria abdomen & $24,54(5,09)$ & $24,94(5,93)$ & 0,373 \\
Adipometria supra-líaca & $24(7,41)$ & $23,6(7,50)$ & 0,750 \\
\hline \% gordura & $30,04(4,74)$ & $30,08(4,40)$ & 0,914 \\
\hline
\end{tabular}

Fonte: Dados da pesquisa.

suprailíaca. Sabe-se que a iontoforese apresenta o potencial de vencer várias limitações associadas à liberação transdérmica, como promover a penetração de moléculas polares e de grande massa molecular (19-22). Simon et al. (23) relatam maior penetração de amitriptilina de $\mathrm{HCl}$ por iontoforese em relação à difusão passiva. Assim, o uso de corrente pode aumentar a liberação transdermal de drogas com baixa permeabilidade, principalmente as hidrofílicas e ionizáveis.

Segundo Frederico et al. (24), a centelha asiática, que foi classificada como droga normalizadora do tecido conjuntivo, agiria na celulite no meio intersticial estimulando a microcirculação, diminuindo o edema, agindo também na redução da gordura localizada.. No entanto, Singh et al. (25) estudaram a transferência por iontoforese de salicilato (ânion), feniletilamina (cátion), manitol (neutro de baixo peso molecular) e insulina (componente neutro de alto peso molecular) usando um modelo de pele humana excisada. Os autores comprovaram que tanto ânions quanto cátions e substâncias neutras de baixo ou alto peso molecular são transferidas por iontoforese com maior efetividade, se comparadas às quantidades transferidas por difusão passiva não influenciada pela passagem da corrente elétrica. A significância encontrada na redução da porcentagem de gordura se dá pelo fato de que sua mensuração ser feita a partir das somas dos valores das dobras cutâneas do tríceps, da região suprailíaca e da região abdominal. A diminuição significativa observada na adipometria de suprailíaca, a leve tendência na adipometria abdominal e os resultados da região do tríceps (dados não apresentados) justificam esses resultados.

No GC, aplicação de cosmético, a diferença significativa foi encontrada na piora dos valores mensurados. Segundo Oliveira et al. (26), de acordo com as características fisiológicas da pele, a transferência transdermal de substâncias ionizáveis por difusão passiva é limitada, principalmente na epiderme. Os resultados do presente estudo sugerem de fato que a aplicação direta do cosmético utilizado neste estudo também não apresenta um efeito importante na redução da gordura localizada. No trabalho de Antony et al. (27), não foi observado efeito anoxerígeno da centelha asiática.

Os resultados da análise estatística intergrupos mostraram que em relação à circunferência da cintura, GA e GB, corrente e iontoforese, respectivamente, apresentaram diferença em relação ao controle $(\mathrm{p}=$ 0,020 ). Na circunferência supraumbilical, GB apresentou uma diferença em relação ao GC $(p=0,017)$ e GD $(p=0,031)$. Na circunferência infraumbilical, GA e GB apresentaram diferença em relação ao GC $(\mathrm{p}=$ 0,002 e p = 0,016) e GD ( $p=0,001$ e p = 0,04). Após a análise estatística, não identificada nenhuma diferença significativa entre GA e GB. No estudo de Carvalho et al. (28), o qual comparou essas duas técnicas no tratamento da dor lombar, não foi observada nenhuma diferença entre elas.

$\mathrm{Na}$ adipometria abdominal, GB apresentou uma diferença em relação ao GD ( $\mathrm{p}=0,021)$; na suprailíaca, GD apresentou uma diferença em relação ao GC (p = $0,006)$. Na porcentagem de gordura, GD apresentou uma diferença em relação ao GA $(p=0,006)$, e GB, 
diferença em relação ao GC (p = 0,017) e ao GD (p $=0,007)$. 0 estudo de Franklin et al. (29) demonstrou que a eletropertubação causada pela aplicação da corrente elétrica pode ser um fator contribuinte para potencializar o transporte do cosmético e por isso a iontoforese pode ser utilizada em tratamentos cosméticos e dermatológicos. No estudo de Zanin et al. (30) detectou-se diminuição da gordura localizada em abdômen e também nos níveis de perfil lipídico, o que mostra que a iontoforese também pode melhorar a saúde dos indivíduos.

\section{Conclusão}

A forma mais eficaz para redução de gordura localizada em flancos é a iontoforese. Esse grupo, GB, apresentou os melhores resultados tanto na comparação intragrupo como na comparação intergrupos. 0 estudo mostra também que a iontoforese tem seus apenas efeitos locais, uma vez que as reduções de medidas ocorreram apenas no local da aplicação, não refletindo em redução de IMC.

\section{Referências}

1. Perozzo G, Olinto M, Costa J, Henn RL, Sarriera J, Pattussi MP. Associação dos padrões alimentares com obesidade geral e abdominal em mulheres residentes no Sul do Brasil. Cad Saúde Públ. 2008;24(10):2427-39.

2. Poli P Neto, Caponi SNC. A medicalização da beleza. Interface. 2007;11(23):569-84.

3. Pitanga FJG, Lessa I. Indicadores antropométricos de obesidade como instrumento de triagem para risco coronariano elevado em adultos na cidade de Salvador - Bahia. Arq Bras Card. 2005;85(1):26-31.

4. Goldenberg M. O corpo como capital: para compreender a cultura brasileira. Arq Mov. 2006;2(2):115-23.

5. Martins EA, Miranda LFD, Sakae TM, Valle LFC. Avaliação de uma série de 38 casos de pacientes submetidos à cirurgia de lipoaspiração em Tubarão-SC entre outubro de 2004 e fevereiro de 2005. Arq Cat Med. 2007;36(1):113-8.

6. Gratieri T, Gelfuso GM, Lopez RFV. Princípios básicos e aplicação da iontoforese na penetração cutânea de fármacos. Quim Nova. 2008;31(6):1490-8.
7. Ferreira AS, Mendonça AC, de Oliveira AI, Martins F, Mazzalli JH, Furtado MA. Estudo da viabilidade da iontoforese na infusão de medicamentos, utilizando eletrodos móveis. Fisioter Bras. 2007;8(6):392-5.

8. Guaratini MI, Oliveira AS, Castro CES. A iontoforese na prática fisioterapêutica. Fisioter Bras. 2007;8(6):430-5.

9. Kumar R, Philip A. Modified transdermal technologies: breaking the barriers of drug permeation via the skin. Trop J Pharm Res. 2007;6(1):633-44.

10. Sreerekha RR, Shanmuga SV, Karthick SP, Srinivas CR, Mathew AC. Study of histamine wheal suppression by dexamethasone with and without iontophoresis. Indian J Dermatol Venereol Leprol. 2006;72(4);283-5

11. Cross SE, Roberts MS. Physical enhancement of transdermal drug application: is delivery technology keeping up with pharmaceutical development?. Curr Drug Deliv. 2004;1(1):81-92.

12. Chorilli M, Brizante AC, Rodrigues CA, Salgado HRN. Aspectos gerais em sistemas transdérmicos de liberação de fármacos. Rev Bras Farm. 2007;88(1):7-13.

13. Milani GB, João SMA, Farah EA. Fundamentos da fisioterapia dermato-funcional: revisão de literatura. Fisiot Pesq. 2006;12(3):37-43.

14. Perini TA, Oliveira GL, Ornellas JS, Oliveira FP. Cálculo do erro técnico de medição em antropometria. Rev Bras Med Esporte. 2005;11(1):81-5.

15. Glaner MF. Índice de massa corporal como indicativo da gordura corporal comparado às dobras cutâneas. Rev Bras Med Esporte. 2005;11(4):243-6.

16. Ferreira AS, Mendonça AC, Oliveira AI, Martins F, Mazzalli JH, Furtado MA. Estudo da viabilidade da iontoforese na infusão de medicamentos, utilizando eletrodos móveis. Fisioter Bras. 2007;8(6):392-5.

17. Bolfe VJ, Guirro R. Resistência elétrica dos géis e líquidos utilizados em eletroterapia no acoplamento eletrodo-pele. Rev Bras Fisioter. 2009;13(6):499-505.

18. Rossoni MA, Nakayama GK, Bertolini GRF. Correntes diadinâmicas de Bernard com e sem iontoforese na DTM: ensaio clínico randomizado. Arq Ciências Saúde. 2009,13(1):3-8.

19. Esteves I Júnior, Tacani PM; Liggieri VC, Ruggi BG, Ferreira LM, Liebano RE. Histamine iontophoresis on the viability of random skin flap in rats. Acta Cir Bras. 2009;24(1):48-51. 
20. Vianna DR, Silva BV, Hamerski L. Eletroporação e iontoforese para liberação de fármacos através da pele. Rev Virtual Quim. 2010 [acesso 20 ago 2013];2(4):271-9. Disponível em: http://www.uff.br/RVQ/index.php/ rvq/article/view/101/143

21. De La Calle JL. Iontophoresis en el tratamiento del dolor neuropático. In: Gozalez-Escalda JR, editor. Iontoforesis: técnica de administración de fármacos. Madrid: Sociedad Española de Dolor; 2001. p 190-202.

22. Fialho SL, Cunha AS Júnior. Iontoforese no transporte ocular de drogas. Arq Bras Oftalmol. 2004;67(5):839-45.

23. Simon L, Weltner AN, Wang Y, Michniak B. A parametric study of iontophoretic transdermal drug-delivery systems. J Memb Sci. 2006;278(1-2):124-32.

24. Frederico MR, Gomes SVC, Melo VC, Martins RB, Lauria $\mathrm{MC}$, de Moura $\mathrm{RL}$, et al. Tratamento de celulite (Paniculopatia Edemato Fibroesclerótica) utilizando fonoforese com substância acoplante à base de hera, centella asiática e castanha da índia. Fisioter Ser. 2006;1(1):6-10.

25. Singh J, Gross M, Sage B, Davis HT, Maibach HI. Effect of saline iontophoresis on skin barrier function and cutaneous irritation in four ethnic groups. Food Chem Toxicol. 2000;38(8):717-26.
26. Oliveira AS, Guaratini MI, Castro CES. Fundamentação teórica para iontoforese. Rev Bras Fisioter. 2005;9(1):1-7.

27. Antony B, Santhakumari G, Merina B, Sheeba V, Mukkadan J.Hepatoprotective effect of Centella asiatica (L) in carbon tetrachloride-induced liver injury in rats. Indian J pharm Scien. 2006;68(6):772-6.

28. Carvalho AR, Fungueto EM, Canzi IM, Barbieiro C, Moraes V, Bertolini GRF, et al. Correntes dinâmicas de Bernard e iontoforese no tratamento da dor lombar. Fisioter mov. 2005;18(4):11-9.

29. Franklin K, Akomeaha D, Gary PM, Marc BB. Shortterm iontophoretic and post-iontophoretic transport of model penetrants across excised human epidermis. Int J Pharm. 2009;367(1-2):162-8.

30. Zanin CTP, Nohama P, Lozz EJ. Efeitos da eletrolipoforese e da iontoforese com cúrcuma no tecido adiposo. In: Anais do 21 Congresso Brasileiro de Engenharia Biomédica, 2005; Salvador. p. 263-6.

Recebido: $15 / 02 / 2012$

Received: 02/15/2012

Aprovado: 17/06/2013

Approved: 06/17/2013 